\title{
Microarray expression profiling and gene ontology analysis of long non-coding RNAs in spontaneously hypertensive rats and their potential roles in the pathogenesis of hypertension
}

\author{
LIANGLEI HOU ${ }^{1}$, ZHENHAO LIN $^{1}$, YUNJIE NI $^{1}$, YIHAO WU ${ }^{1}$, DEZHUN CHEN $^{2}$, LIJUAN SONG $^{1}$, \\ XIAOYAN HUANG ${ }^{3}$, HUANHUAN HU ${ }^{1}$ and DEYE YANG ${ }^{1,3}$
}

\author{
${ }^{1}$ Department of Cardiology, The First Affiliated Hospital of Wenzhou Medical University, Wenzhou, Zhejiang 325000; \\ ${ }^{2}$ Department of Intensive Care Unit, The Second Affiliated Hospital of Wenzhou Medical University, Wenzhou, Zhejiang 325000; \\ ${ }^{3}$ Department of Cardiology, The Affiliated Hospital of Hangzhou Normal University, Hangzhou, Zhejiang 310015, P.R. China
}

Received February 11, 2015; Accepted September 18, 2015

DOI: $10.3892 / \mathrm{mmr} .2015 .4554$

\begin{abstract}
Long non-coding RNAs (IncRNAs) have been demonstrated to be significant in numerous biological processes. Hypertension is a form of cardiovascular disease with at least one billion cases worldwide. The present study sought to compare the differential expression profiles of lncRNAs in the renal cortex of spontaneously hypertensive rats (SHRs) and normotensive Wistar-Kyoto (WKY) rats. The ipsilateral renal cortex was obtained from 15-week-old SHRs and WKY rats whose blood pressures had been monitored. Total RNA was extracted using TRIzol, and lncRNAs and messenger RNAs were profiled by microarray and validated using fluorescent quantitative reverse transcription-polymerase chain reaction. Gene ontology (GO) and Kyoto Encyclopedia of Genes and Genomes (KEGG) analyses were performed to predict the function of differentially expressed genes. Microarray analysis demonstrated that 145 lncRNAs were differentially expressed between SHRs and WKY rats. GO and KEGG pathway analysis indicated that these lncRNAs are involved in numerous biological processes. Thus, lncRNAs may contribute to the pathogenesis of hypertension.
\end{abstract}

Correspondence to: Professor Deye Yang, Department of Cardiology, The Affiliated Hospital of Hangzhou Normal University, 126 Wenzhou Avenue, Hangzhou, Zhejiang 310015, P.R. China E-mail: deyeyang203@hotmail.com

Abbreviations: miRNAs, microRNAs; siRNAs, short interfering RNAs; snRNAs, small nuclear RNAs; ncRNA, non-coding RNA; lncRNA, long non-coding RNA; GO, gene ontology; SHR, spontaneous hypertensive rat; WKY, normotensive Wistar-Kyoto rat

Key words: hypertension, long non-coding RNA, microarray

\section{Introduction}

More than $90 \%$ of the genome is transcribed into non-protein-coding RNAs, including micro- (mi), short interfering (si) and small nuclear (sn) RNAs. Another class of non-coding (nc) RNA, that does not fit into any of these categories, is $>200 \mathrm{nt}$ in length and has been defined as long ncRNA (lncRNA) (1). Recently, lncRNAs have gained widespread attention due to their notable regulatory roles in various pathophysiological changes, including differentiation, proliferation and apoptosis, as well as disease states such as hepatocellular carcinoma and tuberculosis $(2,3)$. Thousands of lncRNA transcripts have been identified in humans, mice and pigs $(2,3)$. Previous studies have indicated that $\operatorname{lncRNAs}$ are highly associated with cardiovascular disease, particularly with regard to cardiac development and myocardial fibrosis $(2,3)$. In addition, certain studies have employed the hypertensive rat model; however, the role of lncRNAs in this type of model remains to be elucidated $(4,5)$.

Hypertension is a cardiovascular disease associated with high morbidity and is increasing in prevalence worldwide (6). Multiple factors are implicated in the pathogenesis of high blood pressure, including the renin-angiotensin-aldosterone system, vascular smooth muscle and vascular endothelial dysfunction, as well as impaired platelet function and kidney-related factors. To further investigate renal-associated hypertension, and taking advantage of recent developments in microarray technology, the present study employed lncRNA microarray to detect and compare differentially expressed ncRNAs in the renal cortex of spontaneously hypertensive rats (SHRs) and normotensive Wistar-Kyoto (WKY) rats.

\section{Materials and methods}

Tissue collection and RNA extraction. SHRs demonstrating increased blood pressure levels at the age of 5-6 weeks, were first obtained by Okamoto and colleagues by inbreeding WKY rats with high blood pressure $(7,8)$. Twelve-week-old SHR and WKY rats (weight, 250-330 g) were obtained from the Experimental Animal Research Center (Zhejiang Chinese Medical University, 
Hangzhou, China) and maintained at $18-29^{\circ} \mathrm{C}, 40-70 \%$ humidity, in a 12/12 h light/dark cycle, 3-5 rats/ cage. Blood pressure levels were monitored using tail cuffs until the rats were 15 -weeks-old (ALCOTT-automated non-invasive blood pressure rat sphygmomanometer; Shanghai Alcott Biotech Co., Ltd., Shanghai, China). All rats were anesthetized with an injection of pentobarbital sodium $(25 \mathrm{mg} / \mathrm{kg}$ ) (Sigma-Aldrich, St. Louis, MO, USA) and perfusion with normal saline prior to the removal of the renal cortex and the heart. All surgical instruments, as well as the operating table, were sterilized using high temperature and high pressure. To remove the renal cortex, an abdominal incision was made, the intestines moved aside and the kidney removed. The kidney was bisected to expose the junction of the medulla and cortex, and the cortex was removed. All of the specimens were flash-frozen in liquid nitrogen and stored in freezing tubes (cat no. 430661; Corning Life Sciences, Corning, NY, USA) at $-80^{\circ} \mathrm{C}$ until use. Three pairs of rats with marked differences in blood pressure were selected as the hypertension and control groups. These were selected, from five pairs of rats with marked differences in blood pressure, following total RNA extraction, as the three selected pairs of samples exhibited the greatest density and purification of RNA. The animal study was approved by the animal welfare committee of Wenzhou Medical University (Wenzhou, China), according to state and institutional regulations.

Total RNA was extracted from the ipsilateral renal cortex (left side) of SHR and WKY rats with TRIzol (Invitrogen Life Technologies, Carlsbad, CA, USA) according to the manufacturer's instructions. The purity and yield of the RNA was evaluated by the ratio of absorbance at $\mathrm{A}_{260}-\mathrm{A}_{280}$ with a NanoDrop 2000 spectrophotometer (Thermo Fisher Scientific, Inc., Wilmington, DE, USA) and 2\% agarose gel electrophoresis (120V, $15 \mathrm{~min}$ ) using 2\% agarose (Biowest, Hong Kong, China), a gel imaging and analysis system (Bio-Rad, Hercules, CA, USA), and an electrophoresis system (Bio-Rad).

lncRNA and mRNA microarray expression profiling and quantitative reverse transcription-polymerase chain reaction $(R T-q P C R)$ validation. The microarray contained $\sim 10,000$ rat lncRNAs that were derived from authoritative databases, including RefSeq (http://www.ncbi.nlm.nih.gov/refseq/), Ensemble and Ultra-conserved Region Encoding LncRNA (https://users.soe.ucsc.edu/ jill/ultra.html), lncRNAdb (http://www.lncrnadb.org/), ncRNA (http://www.ncrna.org/), and sequencing data from the Beijing Aerospace Control Center, which contained 30,367 mRNA probes. Total RNA was reverse transcribed to cDNA using a RevertAid First Strand cDNA Synthesis kit (Thermo Fisher Scientific, Inc., Waltham, MA, USA) with random primers. Following fragmentation, the samples were applied to a custom rat lncRNA microarray, using a LowInput Quick-Amp Labeling kit, Gene Expression Wash kit, Gene Expression Hybridization kit and a RNA Spike-In kit (Agilent Technologies, Inc., Santa Clara, CA, USA). The cDNA fragments were hybridized with the lncRNA microarray chip at $65^{\circ} \mathrm{C}$ for $17 \mathrm{~h}$ according to the manufacturer's instructions. Slides were scanned with the Agilent Microarray Scanner G2505C (Agilent Technologies, Inc.). The data extraction was performed following selection of the appropriate data to export to text, using Feature Extraction software, version 11.0.1.1 (Agilent Technologies, Inc.).
In addition, Feature Extraction software (version 11.0.1.1) was used to analyze the acquired array images of the results. Quantile normalization and subsequent data processing were performed using the GeneSpring GX v12.0 software package (Agilent Technologies, Inc.). Microarray profiling and analysis was conducted by the OE Biotechnology Co. (Shanghai, China).

Six differentially expressed lncRNAs of interest were selected for validation. RT-qPCR was performed using a LightCycler ${ }^{\circledR} 480$ Instrument II (Roche Diagnostics, Basel, Switzerland) with $10 \mathrm{ml}$ PCR reaction mixture (1 ml cDNA, $5 \mathrm{ml} 2$ X LightCycler ${ }^{\circledR} 480$ SYBR Green I Master mix (Roche Diagnostics), $0.2 \mathrm{ml}$ forward primer, $0.2 \mathrm{ml}$ reverse primer and $3.6 \mathrm{ml}$ nuclease-free water). Reactions were incubated in a 384-well optical plate (Roche Diagnostics) at $95^{\circ} \mathrm{C}$ for $10 \mathrm{~min}$, followed by 40 cycles of $95^{\circ} \mathrm{C}$ for $10 \mathrm{sec}$ and $60^{\circ} \mathrm{C}$ for $30 \mathrm{sec}$. Each sample was run in triplicate for analysis. Following the PCR cycles, melting curve analysis was performed specifically to validate the generation of the expected PCR product. The expression levels of sequences were normalized to GAPDH and were calculated according to the $2^{-\Delta \Delta \mathrm{Ct}}$ methodology (9). The primer sequences were designed in the laboratory using Primer 6.0 and Oligo 5.0 and synthesized by Generay Biotech (Shanghai) Co., Ltd. (Shanghai, China) based on the ncRNA sequences obtained from the National Center for Biotechnology Information database (http://www.ncbi.nlm. nih.gov/nucleotide/), as presented in Table I.

Gene ontology (GO) analysis, and functional predictions of $m R N A s$ and $\operatorname{lncRNAs.}$ GO enrichment analysis was used to make preliminary predictions of the biological functions of the sequences of interest. GO is a common method for gene annotation and predominantly includes three fields: Biological processes, cellular components and molecular functions. Kyoto Encyclopedia of Genes and Genomes (KEGG) pathway analysis (http://www.kegg.jp/kegg/docs/statistics.html) is another synthetic pathway prediction tool that contains $>200$ pathways, and a collection of pathway maps representing information based on the molecular interaction and reaction networks for sequences (10).

The final gene list was submitted to the FunNet database (http://www.funnet.ws/) for in-depth analysis of the mRNA or associated lncRNA in the microarray. FunNet is described as an exploratory tool that performs relevance-based searching in transcriptional coexpression networks; the information in FunNet was extracted from genomic databases, converted to a comprehensive exploratory framework and then updated to the website (11). The results provide values to describe the association between the submitted genes and the terms in the database.

\section{Results}

IncRNAs are differentially expressed between SHRs and WKY rats. Microarray analysis indicated that 145 of 9,991 ncRNAs were differentially expressed in SHRs compared with WKY rats; of these, 93 ncRNAs were upregulated and 52 were downregulated ( $\geq 2$-fold; Fig. 1). The included sequences were demonstrated to have functions that predominantly begin with 'NR', such as NR_038078.1, also referred to as small 


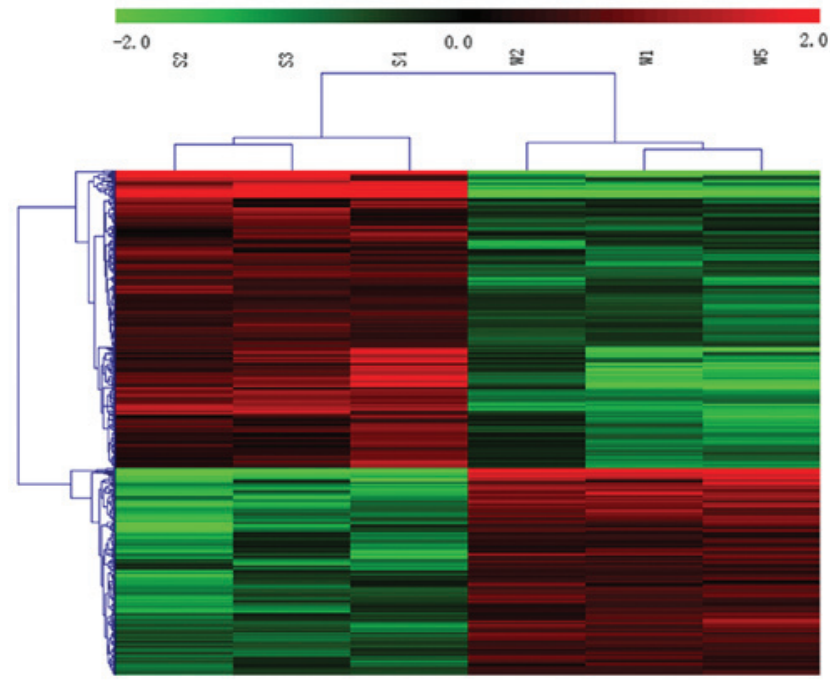

Figure 1. Heat map demonstrating differentially expressed lncRNAs between spontaneously hypertensive rat samples (S2, S3, S4) and Wistar-Kyoto rat samples (W1, W2, W5). Each row represents one lncRNA contained in the microarray and each column represents one tissue sample. The relative lncRNA expression level was downregulated. The red color indicates upregulation. Fold changes are represented by $-2.0,0$ and 2.0 in the corresponding spectrum. lncRNA, long non-coding RNA.

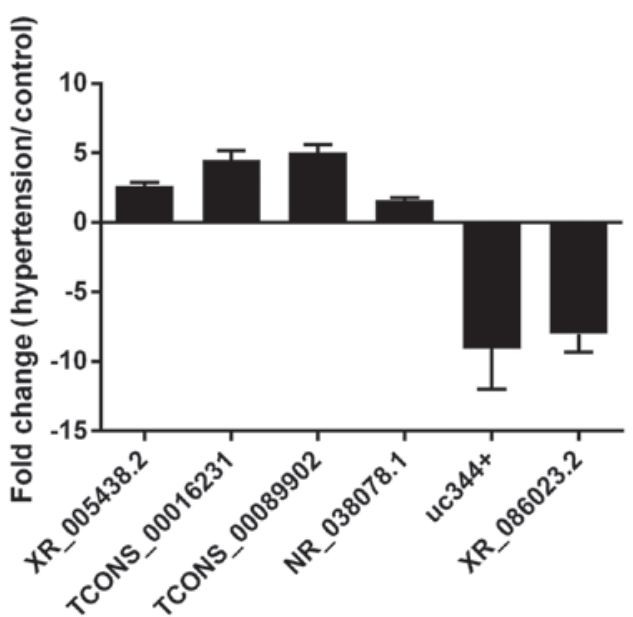

Figure 2. Verification of the expression of six downregulated long non-coding RNAs via quantitative reverse transcription-polymerase chain reaction.

nucleolar RNA host gene 4 (SNHG4), a gene which may be associated with the bystander effect in radiation biology (12). The included sequences were also functionally associated with miscellaneous RNAs, pseudogenes, ultraconserved elements and unclassified sequences (partial data are listed in Table II). Using the same criteria, 383 differentially coexpressed messenger RNAs were identified.

$R T-q P C R$ validation of the differential expression of partial sequences. Six differentially expressed sequences were selected to validate the results of the microarray using RT-qPCR. Partial sequences indicated greater differences, however were not selected due to disparities between groups, for example, TCONS_00031872 demonstrated virtually no expression in the SHR group. The result of RT-qPCR validation is presented in Fig. 2.
Table I. Primer sequences.

\begin{tabular}{ll}
\hline Gene symbol & \multicolumn{1}{c}{ Primer sequence (5'-3') } \\
\hline XR_005438.2 & $\begin{array}{l}\text { TGACTGTAGCTTCACAGGAAT } \\
\text { TCCAGGACAGTTCAGGAT }\end{array}$ \\
TCONS_00016231 & CAAAGTACCTCACCTTACCAG \\
ACTTCCATGACTCTAGCCT \\
TCONS_00089902 & $\begin{array}{l}\text { TTATCGGGAGAGGCTCAAC } \\
\text { GCTACATTGGATCATCTTGTCA }\end{array}$ \\
NR_038078.1 & CTGCTTTGTAAAAGCAAAGGT \\
XR_086023.2 & TCCAAAGACAGTACTAACAAC \\
& AGGTGCCATCGGAGGAATCATCT \\
Uc344+ & CCAATATCCCTGCCTATAACA \\
& TAAGTCCAATCCGCCGTA
\end{tabular}

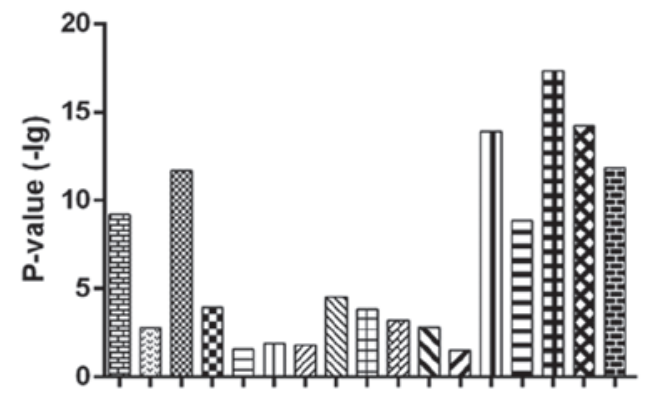

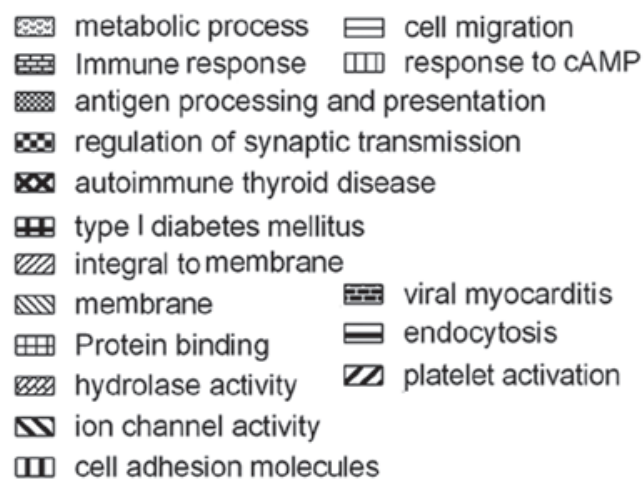

Figure 3. Partial terms of interest or high-scoring terms from GO and KEGG pathway analysis. Seventeen terms that were of interest or had high scores were collected from the GO and KEGG pathway analyses, and integrated into a bar chart; the Y-axis represents the P-value of the statistics provided by the FunNet database. GO, gene ontology; KEGG, Kyoto Encylopedia of Genes and Genomes; cAMP, cyclic adenosine monophosphate.

lncRNAs may serve roles in numerous biological processes and pathophysiological alterations. GO enrichment analysis is based on the knowledge of various biological elements, and KEGG annotations contain information on $>200$ pathways. Certain notable or high-scoring terms in the GO and KEGG pathway analyses were collected in a summary and are listed in Fig. 3. The GO functions identified were predominantly associated with isoprenoid biosynthetic processes, fatty acid metabolic processes, responses to cyclic AMP, cell migration in biological processes, the apical plasma membrane, microsomes, the external side of the plasma membrane, the perinuclear region of the cytoplasm (identified during cellular 
Table II. Partial differentially expressed lncRNAs (abs $\geq 3$-fold) between SHRs and control WKY rats.

\begin{tabular}{|c|c|c|c|}
\hline Target_id & $\mathrm{Chr}$ & Regulation & $\begin{array}{c}\mathrm{FC} \\
\text { (abs) }\end{array}$ \\
\hline TCONS_00016233 & 1 & $\mathrm{Up}$ & 3.03 \\
\hline TCONS_00054808 & 16 & Up & 3.11 \\
\hline XR_085734. 1 & & Up & 3.12 \\
\hline XR_086159. 2 & & Up & 3.13 \\
\hline TCONS_00054032 & 16 & Up & 3.17 \\
\hline TCONS_00110995 & 6 & Up & 3.23 \\
\hline TCONS_00045352 & 14 & Up & 3.25 \\
\hline TCONS_00108373 & 6 & Down & 3.26 \\
\hline TCONS_00058983 & 17 & Up & 3.26 \\
\hline uc. $107+$ & 3 & Down & 3.30 \\
\hline NR_038078. 1 (Snhg4) & 18 & Down & 3.35 \\
\hline XR_086329. 2 & & Up & 3.35 \\
\hline TCONS_00005805 & 1 & $\mathrm{Up}$ & 3.39 \\
\hline TCONS_00045737 & 14 & Down & 3.39 \\
\hline TCONS_00089821 & 3 & Down & 3.41 \\
\hline TCONS_00066490 & 2 & Up & 3.45 \\
\hline XR_146926. 1 & & Down & 3.49 \\
\hline TCONS_00016688 & 1 & Up & 3.53 \\
\hline TCONS_00135006 & 9 & Up & 3.55 \\
\hline TCONS_00045364 & 14 & Up & 3.70 \\
\hline TCONS_00054225 & 16 & Up & 3.75 \\
\hline XR_085687. 1 & & Up & 3.76 \\
\hline XR_147157.1 & & Up & 3.98 \\
\hline TCONS_00077817 & 20 & $\mathrm{Up}$ & 4.01 \\
\hline TCONS_00016690 & 1 & Up & 4.06 \\
\hline TCONS_00043934 & 14 & $\mathrm{Up}$ & 4.07 \\
\hline TCONS_00053182 & 16 & Up & 4.16 \\
\hline TCONS_00003762 & 1 & Up & 4.25 \\
\hline TCONS_00066377 & 19 & Down & 4.30 \\
\hline XR_085881. 1 & & Down & 4.38 \\
\hline TCONS_00134998 & 9 & Up & 4.40 \\
\hline TCONS_00073324 & 2 & Down & 4.41 \\
\hline TCONS_00079171 & 20 & Up & 4.49 \\
\hline TCONS_00041050 & 14 & $\mathrm{Up}$ & 4.68 \\
\hline TCONS_00119346 & 7 & Down & 4.85 \\
\hline TCONS_00063145 & 19 & Up & 4.88 \\
\hline TCONS_00017712 & 10 & Down & 4.93 \\
\hline ENSRNOT00000010509 & 7 & Down & 4.94 \\
\hline TCONS_00016231 & 1 & Up & 5.00 \\
\hline XR_086204. 2 & & Up & 5.03 \\
\hline uc. $101+$ & 3 & Up & 5.43 \\
\hline TCONS_00134930 & 9 & Up & 5.47 \\
\hline TCONS_00058134 & 17 & Up & 6.20 \\
\hline TCONS_00089902 & 3 & Up & 6.36 \\
\hline ENSRNOT00000036466 & $\mathrm{X}$ & Down & 6.39 \\
\hline TCONS_00045074 & 14 & Up & 6.61 \\
\hline TCONS_00045348 & 14 & Up & 6.97 \\
\hline XR_085575. 1 & & Down & 7.38 \\
\hline NR_003722. 1 (Gapdh-ps1) & 3 & Up & 9.81 \\
\hline XR_006589.2 & & Down & 10.49 \\
\hline
\end{tabular}

Table II. Continued.

\begin{tabular}{lccc}
\hline Target_id & Chr & Regulation & $\begin{array}{c}\text { FC } \\
(\text { abs })\end{array}$ \\
\hline XR_006589.2 & & Down & 10.49 \\
TCONS_00106261 & 5 & Down & 11.38 \\
TCONS_00016693 & 1 & Up & 17.88 \\
XR_006738.3 & & Up & 18.33 \\
TCONS_00139993 & X & Down & 27.31 \\
TCONS_00031872 & 11 & Down & 29.43 \\
TCONS_00098342 & 4 & Down & 62.10 \\
\hline
\end{tabular}

Chr, chromosome; lncRNAs, long non-coding RNAs; SHR, spontaneously hypertensive rats; WKY, Wistar-Kyoto; FC, fold change; abs, absolute.

component analysis), glycoprotein binding, phosphatase activity and isopentenyl-pyrophosphate $\delta$-isomerase activity (identified during molecular function analysis). The KEGG pathway analysis demonstrated that lncRNA coexpressed genes predominantly mapped to three pathways, including the cytochrome p450-associated process, extracellular matrix-receptor interaction and viral myocarditis.

\section{Discussion}

These preliminary data indicate that numerous lncRNAs are differentially expressed between SHRs and normal (WKY) rats. These results were expected as high blood pressure (considered to be a multifactorial disease) involves complicated biological networks.

According to the GO and pathway analyses, numerous genes are associated with a variety of membrane functions, particularly including cell membrane permeability, transmembrane receptors and membrane potential. However, this finding contributes little guidance for follow-up experiments, as these physiological elements are involved in almost all biological processes. Although type I diabetes mellitus and viral myocarditis are associated with blood pressure or are involved in the cardiovascular system and received high scores in the pathway analysis, it is challenging to conduct further research on pathways with such a direct association to blood pressure.

One notable finding of the present study was the observation of altered cell migration and ion channel activity, which are two pathogenic factors associated with hypertension. Excessive activation or deactivation of ion channels may have a marked effect on vasomotion or blood pressure, and the target genes of IncRNAs may be associated with the $\mathrm{Na}^{+} / \mathrm{K}^{+}$ATP pump or $\mathrm{Ca}^{+}$channel $(13,14)$. Changes in vascular smooth muscle cell proliferation and migration are considered to be pathogenic factors leading to high blood pressure, as these processes significantly contribute to angiogenesis and microcirculation blood volume (15).

A fundamental limitation of the present study was the disorganized database and unofficial analysis tool used for the identification of animal lncRNAs, including the use of trans- and/or cis-regulatory network analysis. 
Although this is a common method to analyze associated protein-coding genes and transcription factors, as well as to predict biological pathways, these databases are based on the human genome and therefore cannot be used to determine sequence information from rats or mice. The above-mentioned GO and KEGG pathway analyses were used as an effective prediction tool for mRNAs; however, with this approach, the user is capable of predicting only the biological function of coexpressed lncRNAs indirectly. Furthermore, the number of studies and the database integrity for rat lncRNAs are markedly lower than those for human and mouse models. Other biological tools, such as ncFANS Function Annotation (http://www.bioinfo.org/ncfans/), are primarily based on data from humans and mice.

Additional limitations of the present study, as well as other ncRNA studies, are the result of the following factors. First, a number of ncRNAs are shorter than $200 \mathrm{nt}$, are functional, and cannot be grouped into miRNAs and structural RNA groups (16); the microarray in the present study contained only lncRNA probes longer than $200 \mathrm{nt}$. In addition, many ncRNAs are too similar to other mRNAs with regards to sequence. For example, the IncRNA, NR_003722.1 was upregulated in the SHR group in the current results; however, this represents a pseudogene of GAPDH and there is little difference in base sequence composition. Therefore, one of the main obstacles in investigating lncRNAs is designing a primer or siRNA that cannot be associated with similar mRNAs. Furthermore, siRNAs typically interfere with the expression of their target mRNA in the cytoplasm, whereas numerous lncRNAs have been suggested to be located intranuclearly, where the majority of siRNAs have difficulty entering (17). Further to design and synthesize, ensuring the function of the siRNA presents another challenge.

Recently, an RNA sequencing study reported that lncRNAs were differentially altered between the Brown Norway rat and the Dahl salt-sensitive rat, which is recognized as another classical animal model of hypertension. However, Wang et al (4) stated that one limitation of investigating lncRNAs in hypertension is the lack of systematic lncRNA characterization in rats. In another renal lncRNA study that performed sequencing and lncRNA transcriptome analysis, >3,000 transcripts were identified as rat lncRNAs; Gopalakrishnan et al (5) reported numerous lncRNAs that were differentially expressed between Dahl salt-resistant, and Dahl salt-sensitive rats and SHRs. The study also identified that certain mRNAs were coexpressed with lncRNAs and that the majority of lncRNAs were not predicted to have target genes (5). These studies contribute important information to the role of lncRNAs in hypertension in rats.

Previous studies have identified that genes associated with hypertension exert significant influences on lncRNAs. For example, angiotensin II (AngII), a classic hypertensive factor, has been demonstrated to regulate the lncRNA termed Lnc-Ang362 in vascular smooth muscle cells. Following treatment with AngII, the expression level of more than three IncRNAs was dynamically altered; specifically, upregulated lncRNAs increased and downregulated lncRNAs decreased at an early time-point following treatment. However, these levels all returned to baseline within $24 \mathrm{~h}$ (18). The growth of vascular endothelial cells was also indicated to be regulated by the recently described lncRNA, metastasis associated lung adenocarcinoma transcript 1 (MALAT1); in particular, silencing MALAT1 using siRNA was observed to induce various changes in endothelial cells with respect to angiogenesis (19).

Numerous studies have identified miRNAs as highly associated with blood pressure $(13,20)$, and recent studies have highlighted the competing endogenous RNA reaction between miRNAs and lncRNAs. This reaction may represent a novel pathological process, as numerous miRNA-lncRNA or miRNA-mRNA interactions may be implicated in the multilayered lncRNA-mRNA/protein crosstalk (21). Furthermore, these mechanisms have been repeatedly reported for the cardiovascular system (22). In a previous study, two miRNAs (miR-221 and miR-222) were verified to be associated with lncRNA in AngII-treated vascular smooth muscle cells, indicating that lncRNAs may serve as host genes for miRNAs (18). Recently, another study demonstrated the importance of the tripartite axis of IncRNA-miRNA-mRNA in the regulation of cardiovascular disease, which may present as a novel research direction in future (23).

It is not considered to be practical to investigate lncRNAs or miRNAs for the diagnosis of hypertension, as blood pressure measurements may be more readily obtained; however, RNAs may serve as valuable diagnostic tools for individuals with early genetic alterations that do not demonstrate any disease symptoms. For healthy individuals who live with anxiety, psychological stress, or poor living habits and who may be asymptomatic genetic carriers of the hypertensive trait, blood pressure measurements may not indicate abnormalities, whereas molecular biology may provide information on the likelihood of disease. Furthermore, a large number of studies have indicated that miRNAs are closely associated with hypertensive therapy (24). However, the development of essential therapeutic agents utilizing ncRNAs may require significant further research. In conclusion, with the identification of the first miRNA-targeted therapeutic agent and its application in volunteers (25), IncRNAs may present as a novel target for the treatment of hypertension and may potentially serve as molecular therapeutic strategies.

\section{Acknowledgements}

The present study was supported by the National Natural Science Foundation of China (grant no. 81270230) and Ministry of Health of the People's Republic of China Science Foundation (grant no. WKJ-ZJ2014-020).

\section{References}

1. Ponting CP, Oliver PL and Reik W: Evolution and functions of long noncoding RNAs. Cell 136: 629-641, 2009.

2. Schonrock N, Harvey RP and Mattick JS: Long noncoding RNAs in cardiac development and pathophysiology. Circulation Res 111: 1349-1362, 2012.

3. Thum T: Noncoding RNAs and myocardial fibrosis. Nat Rev Cardiol 11: 655-663, 2014.

4. Wang F, Li L, Xu H, Liu Y, Yang C, Cowley AW Jr, Wang N, Liu P and Liang M: Characteristics of long non-coding RNAs in the Brown Norway rat and alterations in the Dahl salt-sensitive rat. Sci Rep 4: 7146, 2014.

5. Gopalakrishnan K, Kumarasamy S, Mell B and Joe B: Genome-wide identification of long noncoding RNAs in rat models of cardiovascular and renal disease. Hypertension 65: 200-210, 2014. 
6. James PA, Oparil S, Carter BL, Cushman WC, Dennison-Himmelfarb C, Handler J, Lackland DT, LeFevre ML, MacKenzie TD, Ogedegbe O, et al: 2014 evidence-based guideline for the management of high blood pressure in adults: Report from the panel members appointed to the Eighth Joint National Committee (JNC 8). JAMA 311: 507-520, 2014.

7. Pinto YM, Paul M and Ganten D: Lessons from rat models of hypertension: From Goldblatt to genetic engineering. Cardiovasc Res 39: 77-88, 1998 .

8. Okamoto K and Aoki K: Development of a strain of spontaneously hypertensive rats. Jpn Circ J 27: 282-293, 1963.

9. Livak KJ and Schmittgen TD: Analysis of relative gene expression data using real-time quantitative PCR and the 2(-Delta Delta C(T)) Method. Methods 25: 402-408, 2001.

10. Kanehisa M and Goto S: KEGG: Kyoto encyclopedia of genes and genomes. Nucleic Acids Res 28: 27-30, 2000.

11. Prifti E, Zucker JD, Clement K and Henegar C: FunNet: An integrative tool for exploring transcriptional interactions. Bioinformatics 24: 2636-2638, 2008.

12. Chaudhry MA: Small nucleolar RNA host genes and long non-coding RNA responses in directly irradiated and bystander cells. Cancer Biother Radiopharm 29: 135-141, 2014.

13. Haddy FJ and Pamnani MB: The vascular $\mathrm{Na}^{+}-\mathrm{K}^{+}$pump in low renin hypertension. J Cardiovasc Pharmacol 6 (Suppl 1): S61-S74, 1984.

14. Bátkai S and Thum T: MicroRNAs in hypertension: Mechanisms and Therapeutic targets. Curr Hypertens Rep 14: 79-87, 2012.

15. Qiu J, Zheng Y, Hu J, Liao D, Gregersen H, Deng X, Fan Y and Wang G: Biomechanical regulation of vascular smooth muscle cell functions: From in vitro to in vivo understanding. J R Soc Interface 11: 20130852, 2014.
16. Spizzo R, Almeida MI, Colombatti A and Calin GA: Long non-coding RNAs and cancer: A new frontier of translational research? Oncogene 31: 4577-4587, 2012.

17. Bagasra $\mathrm{O}$ and Prilliman KR: RNA interference: the molecular immune system. J Mol Histol. 35: 545-553, 2004.

18. Leung A, Trac C, Jin W, Lanting L, Akbany A, Sætrom P, Schones DE and Natarajan R: Novel long noncoding RNAs are regulated by angiotensin II in vascular smooth muscle cells. Circ Res 113: 266-278, 2013.

19. Michalik KM, You X, Manavski Y, Doddaballapur A, Zörning M, Braun T, John D, Ponomareva Y, Chen W, Uchida S, et al: Long noncoding RNA MALAT1 regulates endothelial cell function and vessel growth. Circ Res 114: 1389-1397, 2014.

20. Synetos A, Toutouzas K, Stathogiannis K, Latsios G, Tsiamis E, Tousoulis D and Stefanadis C: MicroRNAs in arterial hypertension. Curr Top Med Chem 13: 1527-1532, 2013.

21. Tay Y, Rinn J and Pandolfi PP: The multilayered complexity of ceRNA crosstalk and competition. Nature 505: 344-352, 2014.

22. Ge D, Han L, Huang S, Peng N, Wang P, Jiang Z, Zhao J, Su L, Zhang S, Zhang Y, et al: Identification of a novel MTOR activator and discovery of a competing endogenous RNA regulating autophagy in vascular endothelial cells. Autophagy 10: 957-971, 2014.

23. Wu C and Arora P: Long noncoding RNA-microRNA-mRNA: A novel tripartite axis in the regulation of cardiac hypertrophy. Circ Cardiovasc Genet 7: 729-731, 2014

24. Neves VJ, Fernandes T, Roque FR, Soci UP, Melo SF and de Oliveira EM: Exercise training in hypertension: Role of microRNAs. World J Cardiol $6: 713-727,2014$

25. Lindow $M$ and Kauppinen S: Discovering the first microRNA-targeted drug. J Cell Biol 199: 407-412, 2012. 